\title{
A Review on Stroke Lesion Analysis in MRI Datasets
}

\author{
Sylvia Irish ${ }^{\mathrm{a}, 1}$, Dhalia Sweetlin $\mathrm{J}$ \\ ${ }^{a}$ Research Scholar, Anna University, Chennai \\ ${ }^{b}$ Dept of IT, MIT Campus, Anna University, Chennai
}

\begin{abstract}
Stroke is one of the major causes of disability in the world. The various disabilities due to stroke includes intellectual functioning damage locomotory dysfunction, visual and hearing impairment, disfigurement etc., This occurs due to occlusion or inhibition of blood supply to the brain which is known as Ischemic stroke, whereas rupturing of blood vessels to the brain causes hemorrhagic stroke. Imaging techniques such as $\mathrm{CT}$ and MRI have played a huge role in the visualization of stroke lesions. Segmentation of the salvageable tissue and its outcome of how it affects a person's lifestyle is the key focus of research as automated model is highly recommended to reduce the time and increase the accuracy in the prediction of stroke lesion in comparison to manual intervention.
\end{abstract}

Keywords. Stroke lesions, Image Modalities-CT, MRI, Segmentation, Convolutional NeuralNetwork.

\section{Introduction}

Cerebro vascular accident (CVA) is the major cause of disability or death across the world.CVA occurs due to occlusion of blood vessel due to atherosclerosis or occurrence of embolus in the blood vessels causes ischemic stroke[1]. High pressure or weak blood vessels rupture causing hemorrhagic stroke where the blood is not supplied to the region beyond the rupture. Necrosis of the brain tissue due to lack of blood flow causes temporary or permanent damage to the person[2]. Treatment for stroke involves identifying the penumbra (potentially recoverable)and core(irrecoverable) regions of the lesion and restoring the blood flow to the former where flow of blood is restricted[3]. Early intervention and accurate detection can cause relatively less damage based on the functional outcome after stroke[4]. NonInvasive modalities like magnetic resonance imaging(MRI) and computerized tomography(CT) are used in the detection of brain lesions. Change in the water molecules of the brain due to the effect of magnetic field produces an increased signal in MRI and reduced signal in CT which helps in the detection of stroke. Manual intervention in identifying the stroke affected area is a cumbersome and tedious task. Thus, various computer aided techniques have been developed in the recent years to support the effective diagnosis of brain tissue damages.

Brain CT provides detailed information along the horizontal or axial view of the head in comparison to X- ray. CT of the brain is performed to assess the injuries,

\footnotetext{
${ }^{1}$ Sylvia Irish, Research Scholar, Anna University, Chennai, India; E-mail: jdsweetlin@annauniv.edu.in
} 
inter-cranial bleeding, lesions and structural anomalies. Radiation exposure is the main disadvantage of $\mathrm{CT}$ as the exposure limit must be consulted with the physician. Similarly, the contrast medium used could cause allergic reactions[5]. MR Imaging of the brain uses magnetic and radio waves to provide detailed structure of the brain. MRI is safer than CT as it does not use radiation [6]. The radio waves manipulate the position of the atoms which is send to the system for producing images. It provides a clear vision of the brain in comparison to CT, X- ray or ultrasound[7]. CT scans are widely used as they are less expensive. However, MRIs produce a detailed image in contrast to CT. Soft tissues, Ligaments and internal organs produce a detailed image in MRI than CT[8].

Image segmentation of the brain has opened new horizons in studying the lesions of the brain for medical diagnostics. Region of Interest (ROI) is obtained through precise segmentation which is a complex and tedious task if performed manually. This paper presents a survey on stroke lesion segmentation and discusses the validation metrics such as accuracy, precision, sensitivity and dice coefficient.The general architecture for stroke lesion analysis system is presented in fig 1 .

\section{Methods and Techniques}

Chen et al.,[9] proposed a method of identifying the white matter hyperintensity of the brain as it is closely associated with stroke, dementia and cognitive impairment. Iterative linearly constrained minimum variance (ILCMV) is a classification method which iteratively captures the spatial information through LCVM which is used to effectively used in classifying both normal and white matter of the brain by combining both spectral and spatial information to detect the white matter hyper intensity (WMH). This method reduces the time of operation in identifying the classes in the brain using different filters such as gaussian, gabor, guided and bilateral filter (BF). Gaussian filter combined with ILCMV showed an accuracy around 95\% along with dice similarity coefficient (DSC) of 0.936 and above.

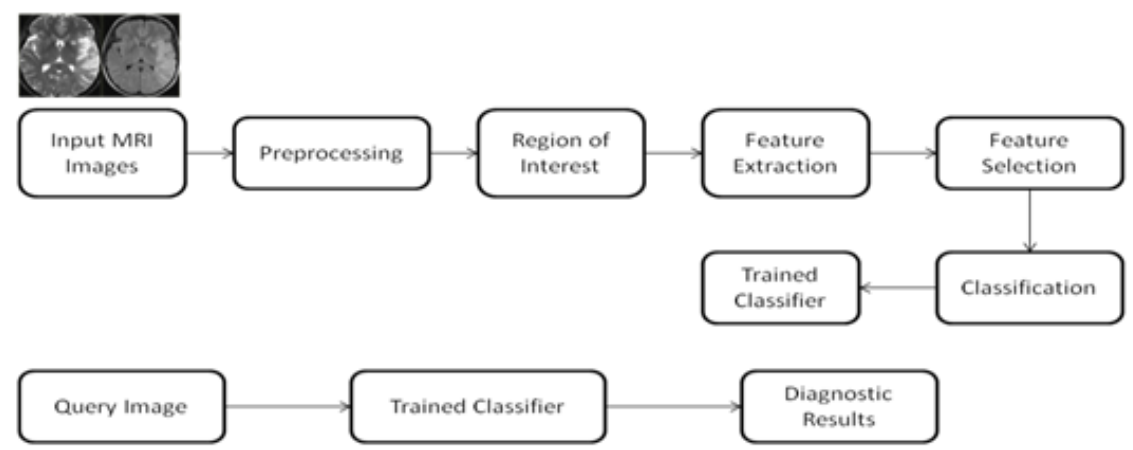

Figure 1., Architecture of general stroke lesion analysis system

Sergio et al., [10] used a method of feature recombination and recalibration where number of feature maps are increased linearly which allows the information to be 
mixed efficiently which is called as spatial adaptive squeeze and excite segmentation (SegSE). The recombination involves mixing the features linearly whereas recalibration is used to obtain the relationship between the features and suppress the less relevant ones. FCN is utilized for the segmentation of lesions. Thesqueeze operation involves suppressing the feature maps along with ReLu activation which is further recalibrated using excitation approach. This approach when evaluated on ISLES 2017 dataset showed an improved dice coefficient score of 0.34 and sensitivity of 0.55 which was higher than the other methods used in evaluation of lesion prediction.

Rongzhao et al., [11] proposed a 3D fully convolutional and densely connected convolution network for automatic and accurate segmentation of ischemic stroke. These densenets uses 20 layers of convolution which allows input from the previous layers to be included in the next layer. Auxillary classification pathway is used to recover the lost images due to down sampling operation. Dice function is used to rectify the class imbalance problems. Skip connections in the network is overcome by deep residual learning. A densenet consists of dense unit, growth rate and dense block. The dense unit reduces the resolution and number of features which causes a large variation in lesion appearance. Introduction of auxiliary pathway over dense unit increases the prediction volume. The automatic segmentation leads to unwanted rejection of samples that are actually lesions. True lesions along the edge can be missed. The DSC of $77.14 \%$ with lesion precision of $89 \%$ is achieved using this model.

Wang et al., [12] utilized a 3D cross hemisphere neighbourhood difference convnet, a CNN methodology for segmentation which utilizes patch-based approach in 3D space. The symmetry of the brain is considered to identify the lesion region by finding the similarity between the images and the cross-hemisphere neighbourhood difference provides robustness to the symmetry. The Dice score of $80 \%$ was achieved using this method.

Vupputuri et al.,[13] proposed a superpixel based segmentation on axial and coronal MR images. Superpixels based clustering are used to improve the speed and accuracy of the lesion segmentation. Hierarchical region splitting method is used to perform pre-processing and the output image is normalized using Z-score. Sobel operator is used to derive the vertical edges and patch wise intensity and region patchwise intensity is compared to determine the hemisphere with lesions. A simple linear iterative clustering method is used on the hemisphere of the brain where the lesion is present which is a superpixel based segmentation. Agglomerative clustering is used to group the lesions together. The clustering method causes over and under estimation of injury. Multi modal and multi view segmentation was not performed using this method.MICCAI challenge dataset demonstrated higher performance with dice similarity score of $81.14 \%$, average sensitivity of $82.32 \%$ and specificity of $93 \%$.

Sathish et al., [14] illustrated an adversarial learning methodology in deep learning for the semantic segmentation of the brain. Fully automated Convolutional neural network is used to estimate the core and penumbra using sequence maps in MRI. Segmentation includes an encoder-decoder similar to VGG11[15] was used for the large-scale image classification. Discriminator uses a batch normalization and 
leaky ReLU activation to determine the non-linearity. Sigmoid activation was also used in the layers of $\mathrm{CNN}$ which determines the cross-entropy loss and turing test discriminator identifies the penumbra of the lesion. Diffusion-Weighted Imaging (DWI), Time to Peak (TTP) and Time to peak of the impulse response (Tmax) information were trained and 3-fold cross validation was performed. Over and under segmentation was considerably reduced. Unified Diffusion and perfusion map the input for deep learning-based feature segmentation to produce accuracy. Limited availability of diffusion and perfusion images causes overfitting of training data. This method was evaluated on ISLES 2015 dataset to obtain a dice score of 0.82 and 0.73 for segmentation of penumbra and core.

Khademi et al., [16] discussed about the white matter lesion segmentation for FLAIR MRI. Partial volume averaging (PVA) is used to compute the volume of the lesions. This generates an image intensity proportional to the tissue which helps in determining the edge information. Fuzzy edge technique was used to group significant and suppress the rest. Thus, normalizing the edge information. Intensity and edge measures were used to denoise the fuzzy edge. Single modality and model free approach was used for classification which caused high correlation to segmentation by experts. PVA performs poor due to bright CSF in ventricles and assigning low $\alpha$ to small lesions. The method showed an accuracy above $98 \%$ in segmenting WML, brain and CSF.

Akshat et al.,[17] proposed a Multisequence network (MSNet) architecture to determine the lesions in the MRI Image. MSNet, a deep neural network combines multi sequence images to differentiate between core and penumbra. The drop out layer is combined with the convolutional layer output to decrease misclassification. MSNet is used to decrease the over and under estimation of lesions in the MRI sequence. Pixel by pixel comparison is used to decrease the load. Core and penumbra segmentation showed a better performance in core in comparison to penumbra when tested on ISLES 2015 dataset with DSC of 0.68 , sensitivity of 0.805 and specificity of 0.99 .

Yanran et al., [18] proposed a deep lesion symmetry convNet for chronic lesion segmentation using MRI. SymROBEX algorithm was used to perform skull stripping which is based on random forest. Histogram equalizer was then applied to provide better contrast. The network construction was mapping of voxels to TI modal. Post processing includes smoothing of lesion images. The Dice similarity coefficient using bilateral voxel improved the performance from 0.63 to 0.78 as compared to unilateral voxel descriptor.

Asit et al., [19] proposed an expectation- maximization (EM) algorithm which was further processed using fractional order Darwinian particle swarm optimization (FODPSO) to increase the accuracy. Maximum likelihood is computed probabilistically on all the features in an unsupervised manner. The expectation step is to obtain the lost data whereas the maximization step determines the best fit. FODPSO is an optimization method used to control the convergence. GLCM method is used to extract the features of the ROI. Random Forest classifier and decision tree are further used to classify the image. RF classifier provides an accuracy of $93.4 \%$ in determining the class labels. 3D volumetric details of the ROI were not used for analysis. 
Keerthana et al., [20] proposed segmentation of stroke based on Fuzzy CMeans method. Fuzzy C- means clustering used for clustering image objects is an unsupervised method used in various fields effectively. Various data point distances are analysed between each pixel and cluster center. The method minimizes the energy using Lagrange multiplier which performs the needed optimization. The segmented portion is used to extract the region of stroke based on edge detection and the size provided the needed details for decision making.

Haocheng et al., [21] combined both CNN and hand- crafted features for the effective segmentation of the stroke images. A Fully Convolutional neural network is used classification with one down sampling along with three up sampling. Intensity, hemispheric intensity between the pixels, first order statistics of volume along with maximum response filters are used as and crafted features which is further applied to classifiers for effective detection stroke.

Karthik et al., [22] combined watershed approach for segmentation of ROI along with discrete curvelet transformation for feature extraction. Segmentation approach limits the neighbourhood drainage as it utilizes drop of water principle which moves from maxima to local minima. Further, discrete curvature transform discretizes the converted gray image from which the curvature coefficients are determined which helps in analysing the mean, variance, standard deviation, skew and kurotosis for analysing the features for its classification using SVM. This method achieved $99.1 \%$ accuracy for 45 clinical datasets.

Liang et al., [23] proposed a method to automatically segment the stroke lesions along with minimizing the number of false positives. The proposed model contains two framework which includes ensemble adapted DeconvNets (EDD Net) and Multiscale Convolutional Label Evaluation Net (MUSCLE Net). The EDD Net achieves an optimal segmentation at all levels and MUSCLE net removes the number of false positives. EDD Net uses the image patches to identify the lesions using DeconvNets which utilizes convolution and pooling layers along with deconvolution and unpooling layers in order to create segmentation probability map. The smaller lesions are then provided to the MUSCLE Net to differentiate the true and false positives. This method relatively reduced the number of false positives and provided 0.67 Dice coefficient and the lesion detection rate is 0.94 .

Kamnitsas et al.,[24] proposed a 3D convolutional neural network for lesion segmentation. This methodology includes CNN includes 11 layers which is sampled in parallel processing of input images through convolutional pathways. The subsampled input patch is obtained from the original image thus providing a large around the image to be processed. Time to analyse the image has been significantly improved. A dice score of $64 \%$ was achieved when tested on ISLES 2015 dataset.

Shubham et al., [25] proposed a patch-based approach with multi scale convolutional layers are used to segment the MRI images as the region containing lesions are less in comparison to the region associated with lesions. The local and global features are acquired effectively from different paths. The binary accuracy of this method is $96 \%$ with a dice score of 0.81 .

Zhiyang et al.,[26] proposed a 2D sliced based segmentation on multi spectral MRI that includes diffusion weighted image, apparent diffusion coefficient and T2-weighted image using residual convolutional network (Res-FCN). Input 
patches are used to evaluate the lesions which is extracted by using sliding window scheme. The features are extracted from the Res block through convolutional layers. The number of false negatives were decreased on evaluation on the testing data due to high accuracy in segmentation with a mean dice coefficient of 0.645 .

Albert et al., [27] proposed segmentation on multi modal MRI using 3D Unet and Res-Net architecture. The residual block with 2 convolution layers is used for down sampling. The input images are analysed as patches to overcome the class imbalance problem due to a contrast variation in healthy and lesion tissues. A random offset value is provided to the lesion tissues to retain them in the final extraction. The method provided better sensitivity with lower Hausdorff distance. This method achieved a DSC of 0.84 and sensitivity of 0.89 .

Jose et al.,[28] proposed a U-Net based architecture for segmentation tasks. The encoding path is split into N- streams for different image modality along with hyper dense connections to improve the information flow with deep supervision along the reduction of over fitting on small training sets. InceptionNet is employed that includes different kernel sizes for estimation of lesions which improved the dice similarity coefficient to 0.635 and considerable reduction in modified Hausdorff distance to $18.64 \mathrm{~mm}$.

Guerrero et al.,[29] proposed a Fully convolution CNN network called uResNet that contains an analysis path to determine the features and synthesis path combines these features with class likelihood for segmentation. This method uses single path to extract the features of the image patch. The Dice score obtained provides a near perfect result in accordance to lesion prediction algorithm (LPA) and Deepmedic. UResnet is also capable of distinguishing different types of lesions effectively. The stroke dice score increased to $40 \%$ with WMH to $70 \%$.

Subbanna et al.,[30] proposed a Bayesian technique to estimate the lesions using the gabor textures which were extracted from the FLAIR image intensities. The Bayesian classification distinguishes between the lesion and non-lesion voxels which is provided as an input customized Markov Random Fields (MRF). The MRF utilizes both the intensities of the voxel along with the intensity of the neighbourhood pixels in order to preserve the boundary of the lesions. This method qualitatively identifies and segments all the lesions with higher accuracy and average dice coefficient of 0.582 using multi- modal MRI datasets

Anjali et al., [31] proposed a random forest method for segmenting the stroke lesions. Histogram based thresholding which is a pixel level image segmentation to segment the region of interest. Random forest reduces the computational cost as the number of features for splitting is reduced. Segmentation accuracy of 3D MRI is increased with average overlap accuracy measuring 0.67 and average hausdorff distance $28.09 \mathrm{~mm}$.

Oskar et al., [32] proposed a fully automated method for segmentation of stroke using extra trees. Extra tree forest are ensemble classifiers which uses a random threshold value for each feature. This method is useful in training uncertain data and is robust to noise. Evaluation on clinical dataset provides dice coefficient to 0.65.A detailed summary is represented in Table 1.

\section{Conclusion}

Various automatic and semi-automatic based methodologies used in segmentation of stroke were discussed. All the techniques discussed above show relatively high 
satisfactory results but further analysis is needed to increase the accuracy, computational speed and precision with the significant decrease in manual intervention. Most of the methods used ISLES dataset for calculation and hence further studies can be carried with clinical dataset with considerably higher MRI images.

Table 1. Methods used in stroke lesion analysis.

\begin{tabular}{|c|c|c|c|c|}
\hline S.No & $\begin{array}{l}\text { Referen } \\
\text { ce } \\
\text { Paper } \\
\text { No. }\end{array}$ & Dataset Used & Methodology & $\begin{array}{l}\text { Performance } \\
\text { Measure }\end{array}$ \\
\hline 1. & [9] & $\begin{array}{l}\text { BrainWeb and } \\
\text { Clinical dataset }\end{array}$ & ILCMV with Gaussian filters & $\begin{array}{l}\text { Accuracy }-95 \% \\
\text { Dice score }-0.936\end{array}$ \\
\hline 2. & [10] & ISLES 2017 & $\begin{array}{l}\text { spatial adaptive squeeze and excite } \\
\text { segmentation (SegSE) }\end{array}$ & $\begin{array}{l}\text { Dice score- } 0.34 \\
\text { Sensitivity-0.55 }\end{array}$ \\
\hline 3. & [11] & $\begin{array}{l}\text { ISLES2015-SSIS } \\
\text { data set }\end{array}$ & 3D FCN & Dice score -0.77 \\
\hline 4. & [12] & Clinical Dataset & 3D Cross hemisphere convnet & Dice score- 0.80 \\
\hline 5. & [13] & MICCAI & Superpixel based segmentation & $\begin{array}{l}\text { Dice Score- } 0.81 \\
\text { Sensitivity- } 82.32 \% \\
\text { Specificity- } 93 \%\end{array}$ \\
\hline 6. & [14] & ISLES 2015 & $\begin{array}{l}\text { adversarial leaming methodology } \\
\text { using FCN }\end{array}$ & $\begin{array}{l}\text { Dice score-0.82 } \\
\text { (penumbra) }\end{array}$ \\
\hline 7. & [16] & Clinical Dataset & Partial Volume Averaging & Accuracy $-98 \%$ \\
\hline 8. & [17] & ISLES 2015 & MSNet & $\begin{array}{l}\text { Dice score- } 0.68 \\
\text { Sensitivity- } 0.805 \\
\text { Specificity-0.99 }\end{array}$ \\
\hline 9. & [18] & Clinical dataset & ConvNet + symROBEX & $\begin{array}{l}\text { Dice score }-0.63 \text { to } \\
0.78\end{array}$ \\
\hline 10. & [19] & Clinical dataset & $\begin{array}{l}\text { EM Algorithm +FODPSO } \\
\text { +GLCM }\end{array}$ & Accuracy- $93.4 \%$ \\
\hline 11. & [22] & Clinical Dataset & $\begin{array}{l}\text { Watershed method }+ \text { discrete } \\
\text { curvelet transformation }\end{array}$ & Accuracy- $99.1 \%$ \\
\hline 12. & [23] & Clinical Dataset & EDD Net + MUSCLE Net & Dice score -0.67 \\
\hline 13. & {$[24]$} & ISLES 2015 & 3D CNN & Dice score -0.64 \\
\hline 14. & [26] & Clinical Dataset & Res-FCN & Dice score- 0.645 \\
\hline 15. & [27] & ISLES 2015 & 3D U-net +Res Net & $\begin{array}{l}\text { Dice score }-0.84 \\
\text { Sensitivity- } 0.89\end{array}$ \\
\hline 16. & [28] & ISLES & U-Net + InceptionNet & Dice score -0.635 \\
\hline 17. & [29] & Clinical Dataset & UResNet & Dice score -0.70 \\
\hline 18. & [30] & Clinical Dataset & $\begin{array}{l}\text { Bayesian Technique + Markov } \\
\text { Random Fields }\end{array}$ & Dice Score -0.582 \\
\hline 19. & [32] & Clinical Dataset & Ensemble Classifiers & Dice Score -0.65 \\
\hline
\end{tabular}

\section{References}

[1] Adamson J, Beswick A, Ebrahim S.Is stroke the most common cause of disability? J Stroke Cerebrovasc Dis. Journal of stroke and cerebrovascular diseases 2004Jul-Aug;13(4):171-7.

[2] C. Warlow, Epidemiology of stroke .The Lancet, vol. 352, pp. S1-S4,1998

[3] Anthony M. Kaufmann, Andrew D. Firlik, MelanieB. Fukui, Lawrence R. Wechsler, CharlesA. Jungries, and Howard Yonas, Ischemic Core and Penumbra in Human Stroke .Volume 30, Issue 1, Pages 93-99, January 1999.

[4] Arunima Kapoor, Krista L. Lanctôt, Mark Bayley, Alex Kiss, Nathan Herrmann, Brian J. Murray, Richard H. Swartz .Good Outcome Isn't Good Enough .Stroke, Volume 48 Issue 6, pp:1688-1690, 2017.

[5] Charles W Schmidt .CT scans: balancing health risks and medical benefits .Environmental health perspectives vol. 120,3 (2012):A118-A121.

[6] Raymond Y. KwongandE. Kent Yucel, Computed Tomography Scan and Magnetic Resonance Imaging .Circulation, Vol 108, Issue 15, October 2003.

[7] M. Sumithra and S. Malathi .A Survey on Medical Image Segmentation Methods with Different Modalitites . International Journal of Engineering Research \& Technology, Volume 6, Issue02,2018.

[8] A E Chang, Y L Matory, A J Dwyer, S C Hill, M EGirton, S M Steinberg, R H Knop, J A Frank, DHyams and J L Doppman, Magnetic resonance imaging versus computed tomography in the evaluation of soft tissue tumors of the extremities . Annals of surgery vol. 205,4 (1987): pp340-348.

[9] Chi-Chang Clayton Chen, Jyh-Wen Chai, Hung- Chieh Chen, Hsin Che Wang, Yung-Chieh Chang, Yi-Ying Wu, Wen-Hsien Chen, Hsian-Min Chen,San-Kan Lee And Chein-I Chang .Iterative Mixed Pixel Classification for Brain Tissues and WMH in MRI .IEEE Access, Volume7,2019.

[10] Sérgio Pereira, Adriano Pinto, Joana Amorim, Alexandrine Ribeiro, Victor Alves, and Carlos A. Silva, .Adaptive Feature Recombination and Recalibration for Semantic Segmentation with Fully Convolutional Networks . IEEE Transactions on Medical Imaging, Vol 38, No 12, December 2019.

[11] Rongzhao Zhang, Lei Zhao, Wutao Lou, Jill M. Abrigo, Vincent C. T. Mok, Winnie C. W. Chu,Defeng Wang, and Lin Shi .Automatic Segmentation of Acute Ischemic Stroke from DWI Using 
3-D Fully Convolutional DenseNets .IEEE Transactions on Medical Imaging, Vol. 37, No. 9, September 2018.

[12] Yan-Ran Wang, Hengkang Wang, Sophia Chen, Aggelos K. Katsaggelos, Adam Martersteck,James Higgins, Virginia B Hill and Todd B Parrish .A 3D Cross-Hemisphere Neighborhood Difference Convnet for Chronic Stroke Lesion Segmentation .2019 IEEE International Conference on Image Processing (ICIP), Taipei, Taiwan, 2019, pp.1545-1549.

[13] A. Vupputuri, S. Dighade, P. S. Prasanth and N. Ghosh, Symmetry determined superpixels for efficient lesion segmentation of ischemic stroke from MRI . 2018 40th Annual International Conference of the IEEE Engineering in Medicine and Biology Society (EMBC), Honolulu, HI, 2018, pp. $742-745$.

[14] Rachana Sathish, Ronnie Rajan, Anusha Vupputuri, Nirmalya Ghosh and Debdoot Sheet, Adversarially Trained Convolutional Neural Networks for Semantic Segmentation of Ischemic Stroke Lesion using Multisequence Magnetic Resonance Imaging,2019 41st AnnualInternational Conference of the IEEE Engineering in Medicine and Biology Society (EMBC), Berlin, Germany, 2019, pp. 1010-1013.

[15] K. Simonyan and A. Zisserman, Very deep convolutional networks for large-scale image recognition,in Int. Conf. Learn. Repr.,2015.

[16] April Khademi, Anastasios Venetsanopoulos and Alan R. Moody, Robust White Matter Lesion Segmentation in FLAIR MRI, IEEE Transactions on Biomedical Engineering, Vol. 59, No. 3, March 2012.

[17] Akshat Gupta,AnushaVupputuri, Nirmalya Ghosh, Delineation of Ischemic Core and Penumbra Volumes from MRI using MSNet Architecture, Conference proceedings: Annual International Conference of the IEEE Engineering in Medicine and Biology Society. IEEE Engineering in Medicine and Biology Society. 2019. 6730-6733. 10.1109/EMBC.2019.8857708.

[18] Yanran Wang, Aggelos K. Katsaggelos, Xue wang and Todd B Parrish, A Deep Symmetry Convnet For Stroke Lesion Segmentation .2016 IEEE International Conference on Image Processing (ICIP), Phoenix, AZ, 2016, pp. 111-115. doi:10.1109/ICIP.2016.7532329.

[19] AsitSubudhi, Manasa Dash and SukantaSabut, Automated Segmentation and classification of brain stroke using expected-maximization and random forest classifier .Biocybernetics and biomedical engineering, Volume40,2019.

[20] S Keerthana and K Sathiyakumar, Brain stroke segmentation using Fuzzy C-means Clustering. International Journal of Computer Applications, Vol. 154, No4, November2016.

[21] Haocheng Shen, SiyamalanManivannan, Roberto Annunziata, Ruixuan Wang, and Jianguo Zhang, "Combination of CNN and Hand-crafted feature for Ischemic Stroke Lesion Segmentation", Proceedings of Ischemic Stroke Lesion Segmentation, October 2016.

[22] R.Karthik and R. Menaka, A multi-scale approach fordetection of Ischemic stroke from brain MR images using discrete curvature transform.Measurement (Elseiver), pp 223-232,2017.

[23] Liang Chen, Paul Bentley and Daniel Rueckert, Fully automatic acute ischemic lesion segmentation in DWI using convolutional neural networks. Neuro Image: Clinical, pp 633-643,2017.

[24] Konstantinos Kamnitsas, Liang Chen, Christian Ledig, Daniel Rueckert, and Ben Glocker, "MultiScale 3D Convolutional Neural Networks for Lesion Segmentation in Brain MRI", Ischemic Stroke Lesion Segmentation Proceedings2015.

[25] Shubham Joshi, Prof. Sonal Gore, Ischemic Stroke Lesion Segmentation by Analyzing MRI ImagesUsing Deep Convolutional Neural Networks.Helix Vol. 8 Issue 5,2018.

[26] Zhiyang Liu, Chen Cao, Shuxue Ding, Tong Han And Sheng Liu, Towards Clinical Diagnosis: Automated Stroke Lesion Segmentation on Multi- Spectral MR Image Using Convolutional Neural Network", IEEE Access Special Section on Big Data Learning and Discovery, October2018.

[27] Albert Clerigues, Sergi Valverde, Jose Bernal, Jordi Freixenet, Arnau Oliver and Xavier Llado, "Acute and sub-acute stroke lesion segmentation from multimodal MRI, in arXiv:1810.133042018.

[28] Jose Dolz, Ismail Ben Ayed, Christian Desrosiers, Dense Multi-path U-Net for Ischemic Stroke Lesion Segmentation in Multiple Image Modalities,4th International Workshop, BrainLes 2018, Held in Conjunction with MICCAI2018.

[29] R. Guerrero, C. Qin, O. Oktay, C. Bowles, L. Chen,R. Joules, R. Wolz, M.C. Valdés-Hernández, D.A. Dickie, J. Wardlaw, D. Rueckert, White matter hyperintensity and stroke lesion segmentation and differentiation using convolutional neural networks. Neuro Image: Clinical, pp 918-934, 2018.

[30] Nagesh K. Subbana, Deepthi Rajashekar, Bastian Cheng, GotzThomalla, Jens Fiehler, Tal Arbel and Nils D. Forkert, Stroke lesion segmentation in FLAIR MRI Datasets using customized Markov random fields, Frontiers in Neurology, May2019.

[31] Anjali Gautam and Balasubramanian Raman, Segmentation of ischemic stroke lesion from 3D MR images using random forest, Multimedia Tools and Applications,2018. 